\title{
Dark Triad Dirty Dozen: Avaliando seus Parâmetros Via TRI
}

\author{
Emerson Diógenes de Medeiros - Universidade Federal do Piauí \\ Renan Pereira Monteiro - Universidade Federal do Pianí \\ Rildésia Silva Veloso Gouveia - Centro Universitário de João Pessoa \\ Bruna da Silva Nascimento - University of Bath \\ Valdiney Veloso Gouveia - Universidade Federal da Paraíba
}

\begin{abstract}
Resumo
O presente estudo objetivou explorar os parâmetros psicométricos da Dirty Dozen por meio da Teoria de Resposta ao Item (TRI), utilizando o Modelo de Resposta Graduada. Participaram 284 pessoas, com média de idade de 24,2 anos $(D P=8,76)$. Inicialmente, checou-se a dimensionalidade da escala, sendo encontrados resultados que apoiam uma estrutura composta por três fatores oblíquos $(\mathrm{CFI}=0,99$; TLI $=0,98$; RMSEA $=0,03)$. Portanto, as análises de TRI foram realizadas separadamente para cada fator. Posteriormente, verificou-se que os itens discriminam adequadamente os participantes, sendo aqueles que pertencem ao fator narcisismo os mais endossados; maquiavelismo e psicopatia exigiram uma quantidade maior de theta para haver concordância com o conteúdo do item, quiçá em virtude desses traços serem socialmente indesejáveis. Estima-se que os objetivos do estudo foram alcançados, conhecendo evidências dessa medida breve a partir da TRI, referendando seu uso em possibilidades futuras.

Palavras-chave: tríade sombria, maquiavelismo, psicopatia, narcisismo, teoria de resposta ao item
\end{abstract}

Dark Triad Dirty Dozen: Assessing its Parameters Through IRT

\begin{abstract}
The present study aimed to explore the psychometric parameters of the Dirty Dozen through the Item Response Theory (IRT), using the Graduated Response Model. Participants included 284 individuals, with a mean age of 24.2 years $(S D=8.76)$. Initially the dimensionality of the scale was checked and results that support a three-oblique-factor structure were found (CFI $=0.99 ; \mathrm{TLI}=0.98 ; \mathrm{RMSEA}=0.03)$. Therefore, IRT analyses were performed separately for each factor. Then, it was verified that the items discriminate participants properly, being those items belonging to the narcissism factor the ones mostly endorsed; Machiavellianism and psychopathy demanded a greater amount of theta in order to agree with the item content, perhaps because these traits are socially undesirable. It is considered that the aims of the study were achieved, providing evidence of this short measure through the IRT, showing the possibilities of its use in future studies.
\end{abstract}

Keywords: Dark Triad; Machiavellianism; psychopathy; narcissism; Item Response Theory.

\section{Dark Triad Dirty Dozen: Evaluando sus Parámetros Vía TRI}

\begin{abstract}
Resumen
Este estudio tuvo como objetivo explorar los parámetros psicométricos del Dirty Dozen por medio de la Teoría de Respuesta al Ítem (TRI), utilizando el Modelo de Respuesta Graduada. Participaron 284 personas con edad promedio de 24,2 años (DP $=8,76)$. Inicialmente se verificó la dimensionalidad de la escala, encontrándose resultados que apoyan una estructura de tres factores oblicuos (CFI $=0,99$; TLI $=0,98$; RMSEA $=0,03$ ). Por lo tanto los análisis de TRI fueron realizados separadamente para cada factor. Después se verificó que los ítems discriminan adecuadamente los participantes, siendo aquellos que pertenecen al factor narcisismo más endosados; maquiavelismo y psicopatía exigieron una cantidad mayor de theta para que existiese concordancia con el contenido del ítem, quizás debido a que esos rasgos son socialmente indeseables. Se estima que los objetivos del estudio fueron alcanzados, conociendo evidencias de esta medida breve a partir de la TRI, apoyando su uso en posibilidades futuras.

Palabras-clave: Tríada sombría; maquiavelismo; psicopatía; narcisismo; Teoría de Respuesta al Ítem.
\end{abstract}

Nos últimos anos, um agrupamento de traços aversivos da personalidade tem ganhado destaque, sobretudo após o estudo de Paulhus e Williams (2002), que nomearam esse cluster como dark triad of personality (tríade sombria da personalidade), formados por maquiavelismo e pelas formas subclínicas de psicopatia e narcisismo. Portanto, o surgimento desse agrupamento de traços aversivos vem suprir uma lacuna existente na literatura, que contemplava mais os aspectos positivos da personalidade em detrimento daqueles mais sombrios, que, apesar da natureza indesejável, apresentam variações mais brandas que podem fazer parte de uma faixa normal de funcionamento (Huang \& Liang, 2014; Jonason \& Middleton, 2015).

Nessa direção, percebe-se que o foco da tríade sombria está na descrição de pessoas que possuem estratégias comportamentais e tendências psicológicas malévolas (O’Boyle, Forsyth, Banks, Story, \& 
White, 2014). Assim, traços psicopáticos estão relacionados à baixa empatia, impulsividade e busca de sensações; os narcisistas são mais direcionados para aspectos como egocentrismo e grandiosidade; e os maquiavélicos voltam-se para o uso de estratégias de manipulação interpessoal na exploração dos demais, visando a obtenção de ganhos pessoais (Book, Visser, \& Volk, 2015; Jones \& Paulhus, 2011; Patrick, Fowles, \& Krueger, 2009).

Apesar de socialmente ofensivos, tais traços não são restritos a amostras clínicas e em situação prisional, havendo evidências que estes também estão presentes na população geral (Foster \& Campbell, 2007; LeBreton, Binning, \& Adorno, 2006), sendo que as pessoas podem ser situadas, em função de seu traço latente, ao longo de um contínuo. Assume-se, então, que o lado sombrio da personalidade é entendido a partir de um ponto de vista pseudopatológico, capturando a variante subclínica dos construtos, que são vistos como socialmente prejudiciais, atribuindo benefícios imediatos ao invés do bem comum (Book et al., 2015; Jonason, Duineveld, \& Middleton, 2015).

Mesmo com evidências empíricas sugerindo que personalidades sombrias podem predizer emoções e comportamentos sombrios (Giammarco \& Vernon, 2014; Kavanagh, James, Jonason, Chonody, \& Scrutton, 2014), esses traços também podem cumprir funções adaptativas. De fato, estudos no âmbito da Psicologia Evolucionista têm indicado que pessoas com traços socialmente aversivos da personalidade buscam novas relações, preferencialmente aquelas de curto prazo, mesmo com relacionamentos em curso, resultando em um número maior de parceiras sexuais (Jonason, Li, \& Buss, 2010; Jonason, Li, Webster, \& Schmitt, 2009), além de serem vistos como mais atraentes (Carter, Campbell, \& Muncer, 2014). Tais estudos vêm auxiliando na desvinculação da tríade sombria de uma visão estritamente patológica ou disfuncional, indicando que esse agrupamento pode ter um lado adaptativo, auxiliando no sucesso reprodutivo dos indivíduos (Jones \& Figueredo, 2013).

Tendo em vista o crescimento e centralidade que esse conjunto de traços tem tido atualmente, auxiliando no entendimento de uma série de fenômenos, pesquisadores vem buscando conhecer o núcleo do lado sombrio da personalidade, contudo, ainda não há consenso sobre o que causaria a sobreposição dos construtos. Por exemplo, estudos têm indicado que o polo oposto do traço amabilidade (antagonismo) no modelo dos cinco grandes fatores pode ser o aspecto central dos três componentes da tríade (Jakobwitz \& Egan, 2006; Paulhus \& Williams, 2002); por outro lado, há evidências que sugerem que o núcleo comum seria baixos escores no fator $\mathrm{H}$ do modelo HEXACO, havendo, inclusive, evidências empíricas que apoiam esta hipótese (Book et al., 2015). Alternativamente, Jones e Figueredo (2013) propuseram que o aspecto central do lado sombrio da personalidade seria a confluência entre manipulação interpessoal e insensibilidade, sendo que dessa interação emergiriam os traços antagônicos. Outro entendimento é dado por Paulhus (2014), citando que a sobreposição seria em função do traço callous (baixa empatia).

Não obstante à busca pelos aspectos compartilhados, observa-se que os construtos que formam a tríade sombria da personalidade apresentam especificidades. Considerando, por exemplo, o traço callous (baixa empatia), observa-se que ele pode exercer papéis distintos nos três fatores (Paulhus, 2014). A baixa empatia em narcisistas pode ser um fator preponderante para que eles, em busca de reconhecimento, passem por cima dos demais; já a baixa empatia em maquiavélicos pode ser uma vantagem ao procurar pessoas a quem podem explorar; e, por fim, psicopatas fazem o que for preciso para obter o que querem, mesmo que isso implique no sofrimento alheio (Jonason \& Middleton, 2015; Paulhus, 2014).

A partir do exposto, verifica-se a profusão de estudos sobre o lado sombrio da personalidade, buscando conhecer similaridades e diferenças de cada componente da tríade, bem como verificar a contribuição desse agrupamento para o entendimento de comportamentos diversos. Contudo, uma limitação ressaltada por Paulhus (2014) se refere ao tempo gasto quando se está avaliando personalidades sombrias, pois estas têm sido tradicionalmente mensuradas por meio de inventários específicos, dificultando, portando, a avaliação simultânea. Felizmente, nos últimos anos, o panorama tem se modificado com a proposição de instrumentos concisos que avaliam esses construtos sob uma mesma escala de resposta, com destaque para a Dark Triad Dirty Dozen (DTDD; Jonason \& Webster, 2010) e a Short Dark Triad (SD-3; Jones \& Paulhus, 2014).

Concretamente, a estimação desses traços sombrios por meio de medidas curtas tem se popularizado, tal como se pode constatar analisando a edição da revista Personality and Individual Differences, em seu volume 67, de setembro de 2014, dedicado exclusivamente a essa constelação de traços socialmente aversivos da personalidade. Do total dos 21 artigos publicados, foram considerados apenas aqueles que operacionalizaram a 
tríade sombria, sendo que cerca de $60 \%$ a fizeram por meio de medidas curtas (SD3 ou DTDD).

Apesar da popularização da temática e dos mais de quinze anos do estudo inicial de Paulhus e Williams (2002), pouco se tem discutido sobre o lado sombrio da personalidade no contexto brasileiro. Portanto, pensa-se que um passo inicial que pode contribuir para o avanço dessa temática no Brasil seja checar, inicialmente, a qualidade de medidas que operacionalizam esse conjunto de fatores, permitindo a adequada mensuração em estudos futuros. Especificamente nesse ponto, centra-se o presente estudo, que busca evidências psicométricas, a partir da Teoria de Resposta ao Item (TRI), da Dark Triad Dirty Dozen (DTDD), a medida concisa mais empregada para a estimação da tríade sombria da personalidade (Jonason \& Middleton, 2015).

A DTDD foi proposta por Jonason e Webster (2010), sendo um instrumento curto, composto por apenas 12 itens, quatro por fator. Estes autores encontraram, por exemplo, indicadores que atestam a adequação da estrutura de três fatores oblíquos (e.g., CFI > 0,90; RMSEA < 0,10), além de resultados que atestam validade convergente com os instrumentos longos que avaliam separadamente cada construto. Em outros estudos (Jonason, Kaufman, Webster, \& Geher, 2013; Jonason \& Luévano, 2013), modelos alternativos têm sido propostos, havendo evidências em favor do bifator (e.g., CFI > 0,90; NFI > 0,95), sugerindo que, apesar de compartilharem características, os fatores da tríade apresentam especificidades.

Webster e Jonason (2013) também verificaram evidências da DTDD à luz da Teoria de Resposta ao Item (TRI), onde os 12 itens discriminaram as pessoas ao longo do traço latente avaliado. No que se refere à dificuldade, em geral os itens de narcisismo exigiram um valor menor de theta $(\theta)$ para serem endossados, talvez indicando que esse seja o traço mais reluzente entre aqueles que compõem o lado sombrio (Rauthman \& Kolar, 2012). Por outro lado, aqueles que formam o fator psicopatia foram os mais difíceis, quiçá em virtude de sua natureza socialmente indesejável (Webster \& Jonason, 2013).

Por facilitar os estudos sobre a tríade sombria e por apresentar evidências de sua adequação psicométrica em diferentes contextos e com abordagens estatísticas distintas, Gouveia, Monteiro, Gouveia, Athayde e Cavalcanti (2016) decidiram adaptar a DTDD para o Brasil. Esses autores basearam-se na Teoria Clássica dos Testes (TCT), verificando, ao longo de dois estudos, o poder discriminativo dos itens, a confiabilidade dos fatores, a estrutura trifatorial por meio de uma Análise de Componentes Principais e a adequação desta por meio de uma Análise Fatorial Confirmatória, contudo, com indicadores de ajuste inferiores (GFI $=0,92$; TLI $=0,90$; RMSEA $=0,08)$ ao modelo bifator, que se mostrou o mais adequado (e.g. GFI $=0,95$; $\mathrm{TLI}=0,92$; RMSEA $=0,07)$.

O estudo de Gouveia et al., constituiu-se como evidências preliminares sobre a validade fatorial e consistência interna da DTDD em contexto brasileiro. Não obstante, demanda-se o conhecimento de novas evidências, sendo que o presente estudo objetiva avaliar os parâmetros dessa medida breve por meio da TRI.

\section{Método}

\section{Participantes}

Participaram deste estudo 284 voluntários que responderam ao instrumento por meio de um link disponibilizado na internet. Estes tinham idade média de 24,2 anos $(D P=8,76)$, em maioria mulheres $(69,4 \%)$, solteiros $(57,4 \%)$ e que se autodeclararam de classe média $(49,3 \%)$.

\section{Instrumentos}

Dark Triad Dirty Dozen (DTDD; Jonason \& Webster, 2010): Esse instrumento é composto por 12 itens divididos, equitativamente, em três dimensões: Maquiavelismo (e.g., item 3. "Costumo bajular os outros para conseguir o que quero.”), psicopatia (e.g., item 7. "Eu tendo a ser insensível ou indiferente.") e narcisismo (e.g., item 10. "Eu tendo a querer que os outros prestem atenção em mim.”). Aqui se utilizou da versão validada, em português brasileiro, por Gouveia et al., (2016), respondida em escala tipo Likert de cinco pontos $(1-$ Discordo totalmente a 5 - Concordo totalmente).

Por fim, os participantes foram solicitados a preencher um questionário demográfico (e.g., sexo, idade, estado civil e classe social), com finalidade de caracterização da amostra.

\section{Procedimento}

A coleta de dados aconteceu por meio da internet, onde foi divulgado, nas redes sociais, um link que continha o questionário. Destaca-se que foi utilizada a técnica bola de neve, solicitando aos respondentes que, ao finalizarem o preenchimento, divulgassem o link do questionário entre seus contatos. Para ter acesso ao instrumento era necessário indicar ser maior de 18 anos e concordar com o Termo de Consentimento Livre e Esclarecido 
(TCLE). As instruções de como responder aos instrumentos eram autoexplicativas, o caráter voluntário e anônimo do estudo foi destacado, inclusive apontando a possibilidade de desistência da participação a qualquer momento sem qualquer ônus. Assevera-se, portanto, que foram seguidas as recomendações da Resolução CNS 466/12. Em média, as pessoas levaram 15 minutos para finalizar sua participação.

\section{Análise de Dados}

Os programas IBM SPSS e $\mathrm{R}$ (versões 20 e 3.1.3, respectivamente) foram empregados inicialmente. Com o primeiro, foram calculadas as análises descritivas e de confiabilidade, enquanto que com o segundo fora empregado para efetuar as análises fatoriais confirmatórias (AFC), utilizando o pacote Lavaan (Rosseel, 2012). Os indicadores a seguir foram tidos em conta para avaliar a adequação do modelo aos dados (Browne \& Cudeck, 1993; Hair, Black, Babin, Anderson, \& Tatham, 2009; Hu \& Bentler, 1999; Pilati \& Laros, 2007):

Comparative Fit Index (CFI). Esse é um indicador adicional de ajuste do modelo, servindo para comparar com modelos alternativos. Seus valores variam de 0 (zero; ajuste nulo) a 1 (um; ajuste perfeito), admitindo-se os valores superiores a 0,90 como indicativo de ajuste aceitável.

Tucker - Lewis Index (TLI). Esse índice compara o modelo estimado com um modelo teórico nulo, ou seja, visa determinar se todos os indicadores são associados a um único fator latente (Hair et. al., 2009). Valores superiores a 0,95 indicam um ajuste robusto.

Root-Mean-Square Error of Approximation (RMSEA). Esse indicador, com seu intervalo de confiança de 90\% (IC 90\%), testa os residuais entre o modelo teórico estimado e os dados empíricos obtidos. Valores altos são indicativos de um modelo não ajustado, recomendando-se valores próximos ou inferiores a 0,05; 0,08 é um valor comumente aceito, admitindo-se até 0,10 como um modelo aceitável.

Para comparar os modelos, de três fatores com o unifatorial, foram considerados os indicadores de ajuste ora apresentados, sendo penalizado o que apresentar mais pobre ajuste aos dados.

Por fim, utilizou o software R (R Core Team, 2015) para avaliar os parâmetros dos itens por meio da Teoria de Resposta ao Item (TRI). Especificamente, considerando que os itens são politômicos, recorreu-se ao Modelo de Resposta Graduada (Samejima, 1969), utilizando-se, para tanto, o pacote ltm (Rizopoulos, 2006).

\section{Resultados}

Inicialmente, tendo em vista o pressuposto da unidimensionalidade para se realizar a análise dos itens pela TRI, checou-se a estrutura fatorial da DTDD. Optou-se por realizar uma AFC, comparando o modelo com três fatores oblíquos ao unifatorial. Nesse caso, adotou-se o método de estimação dos Mínimos Quadrados Ponderados Robustos (WLSMV - Mean and Variance Adjusted Wighted Least Squares).

O modelo com três fatores inter-relacionados apresentou indicadores que atestam sua adequação psicométrica: $\mathrm{CFI}=0,99$; TLI $=0,98$ e RMSEA (IC90\%) $=0,03(0,01-0,05)$. Verificou-se, ainda, que todas as saturações (lambdas) foram estatisticamente diferentes de zero $(\lambda \neq 0 ; z>1,96, p<0,05)$, apresentando um valor médio de $0,66(D P=0,08)$, variando de 0,50 (Item 5. "Eu tendo a ter falta de remorso.") a 0,78 (Item 9. Eu tendo a querer que os outros me admirem e Item 10. Eu tendo a querer que os outros prestem atenção em mim), como pode ser atestado na Tabela 1 . Por outro lado, o modelo unidimensional apresentou índices de ajuste insatisfatórios: $\mathrm{CFI}=0,86$; TLI $=0,83$ e RMSEA (IC $90 \%)=0,11(0,10-0,12)$. Com evidências de uma estrutura composta por três fatores, as análises de TRI foram realizadas separadamente para cada fator.

\section{Parâmetros dos Itens com a Teoria de Resposta ao Item (TRI)}

Antes de verificar a dificuldade e discriminação de cada item, avaliou-se a fidedignidade de cada subescala da DTDD, por meio do coeficiente alfa de Cronbach $(\alpha)$ e da correlação média inter-itens $\left(r_{\text {mi }}\right)$, sendo que os resultados indicaram valores aceitáveis [maquiavelismo $\left(\alpha=0,73 ; r_{\mathrm{mi}}=0,42\right) ;$ psicopatia $\left(\alpha=0,71 ; r_{\mathrm{mi}}=0,38\right)$; e narcisismo $\left(\alpha=0,83 ; r_{\mathrm{mi}}=0,55\right)$, reforçando a decisão de avaliar separadamente os fatores. Posteriormente, analisou-se a dificuldade, discriminação e a quantidade de informação psicométrica proporcionada pelos itens nos três fatores. Tais resultados são sumarizados na Tabela 1.

Tal como pode ser observado na Tabela 1, percebe-se que todos os itens se mostraram discriminativos, sendo que os valores desse parâmetro foram iguais ou acima de 1,45 . Portanto, os itens possuem a capacidade de diferenciar pessoas em função do respectivo theta $(\theta)$ no traço latente em questão. Especificamente, no que concerne ao fator Maquiavelismo, a média de discriminação foi de 2,00 (DP = 0,37), indo de 1,74 (item 4 . "Costumo explorar outras pessoas para meu benefício próprio.") a 2,53 (item 2. "Costumo usar enganações 
Tabela 1

Lambdas, Discriminação, Dificuldade e Informação dos Itens e Subescalas

\begin{tabular}{lccccccc}
\hline & $\lambda$ & $\mathrm{a}$ & $\mathrm{b}_{1}$ & $\mathrm{~b}_{2}$ & $\mathrm{~b}_{3}$ & $\mathrm{~b}_{4}$ & $\mathrm{I}(\theta ;-3 /+3)$ \\
\hline Maquiavelismo & & & & & & & 16,54 \\
Item 1 & 0,68 & 1,98 & $-0,24$ & 0,51 & 1,13 & 2,32 & 4,56 \\
Item 2 & 0,66 & 2,53 & 0,49 & 1,39 & 2,31 & 2,86 & 5,88 \\
Item 3 & 0,63 & 1,76 & 0,45 & 1,27 & 1,97 & 2,96 & 3,28 \\
Item 4 & 0,62 & 1,74 & 0,92 & 1,81 & 2,71 & 3,64 & 2,81 \\
Psicopatia & & & & & & & 13,57 \\
Item 5 & 0,50 & 1,46 & 0,50 & 1,22 & 2,03 & 3,00 & 2,40 \\
Item 6 & 0,62 & 1,66 & 0,78 & 1,48 & 2,18 & 2,71 & 2,72 \\
Item 7 & 0,63 & 2,36 & 0,08 & 0,68 & 1,34 & 2,70 & 5,66 \\
Item 8 & 0,69 & 1,55 & 0,26 & 1,08 & 2,13 & 3,10 & 2,79 \\
Narcisismo & & & & & & & 29,46 \\
Item 9 & 0,78 & 3,79 & $-1,03$ & $-0,51$ & 0,27 & 1,06 & 11,83 \\
Item 10 & 0,78 & 3,20 & $-1,08$ & $-0,23$ & 0,53 & 1,40 & 10,08 \\
Item 11 & 0,74 & 1,95 & $-0,81$ & $-0,12$ & 0,67 & 1,60 & 4,64 \\
Item 12 & 0,68 & 1,45 & $-0,54$ & 0,37 & 1,26 & 2,45 & 2,90 \\
\hline
\end{tabular}

Nota. $\lambda=$ lambda; $a=$ discriminação; $b_{1-4}=$ dificuldade; $I(\theta ;-3 /+3)=$ Informação no intervalo de -3 a +3
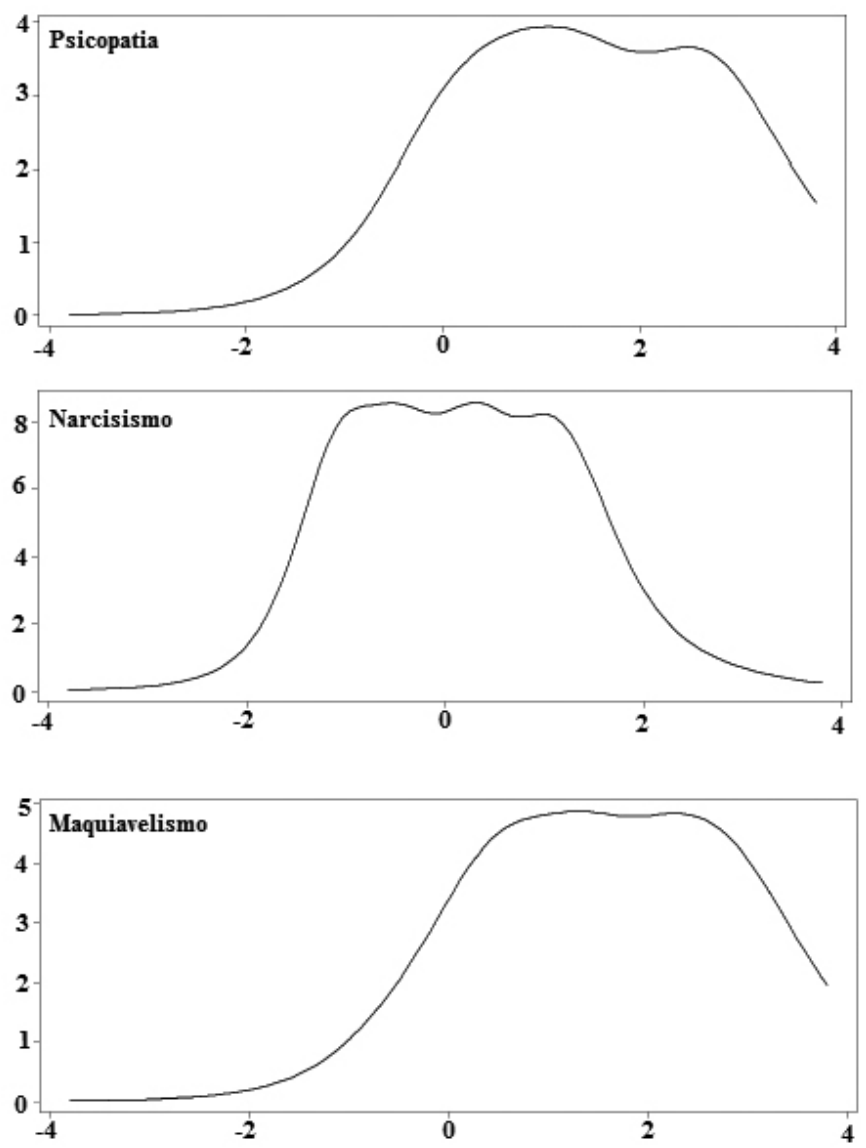

Figura 1. Curva de Informação de cada fator. 
ou mentiras para conseguir o que quero.”). No geral, essa subescala ofereceu 20,36 de informação, sendo que $16,54(81,2 \%)$ encontrou-se no intervalo de -3 a +3 , sendo o item 2 o mais informativo $[\mathrm{I}(\theta ;-3 /+3)=5,88]$. Já para o fator Psicopatia, observou-se uma média de discriminação de 1,75 (DP $=0,41)$, variando de 1,46 (item 5. "Eu tendo a ter falta de remorso.") a 2,36 (item 7. "Eu tendo a ser insensível ou indiferente."). Essa dimensão ofereceu de informação 16,64, sendo que 13,57 (81,56\%) encontrou-se no intervalo de -3 a +3 , sendo o item 7 o mais informativo $[\mathrm{I}(\theta ;-3 /+3)=5,66]$. Por fim, verificou-se que os itens que compõe Narcisismo tiverem uma discriminação média de 2,60 (DP $=1,08$ ), variando de 1,45 (item 12. "Costumo esperar favores especiais dos outros.") a 3,79 (item 9. Eu tendo a querer que os outros me admirem). Essa subescala ofereceu de informação 30,04, sendo que 29,46 (98,06\%) encontrou-se no intervalo entre -3 e +3 , com o item 9 sendo o mais informativo $[\mathrm{I}(\theta ;-3 /+3)=11,83]$.

Mais informações acerca da amplitude de theta que os fatores da tríade cobrem podem ser observadas na Figura 1, que indica que os fatores Maquiavelismo e Psicopatia cobrem mais o intervalo entre 0 e 4 , sendo mais eficazes na discriminação de pessoas que apresentam níveis médios e elevados de traço latente. Por outro lado, Narcisismo é mais informativo na faixa próxima de $-1,5$ a 1,5 , ou seja, é útil para avaliar pessoas com níveis intermediários de theta, contudo, pode ser ineficaz em diferenciar pessoas com thetas mais elevados em função da ausência de itens mais difíceis.

No que tange ao parâmetro de dificuldade e avaliando os limiares de respostas dos três fatores, percebe-se que os itens relativos ao Narcisismo foram os que exigiram uma menor quantidade de theta para serem completamente endossados $(M=1,62 ; D P=$ 0,59 ), sendo o item 12 ("Costumo esperar favores especiais dos outros."), aquele que exigiu maior quantidade do traço para haver total concordância $\left(b_{4}=2,45\right)$. Por outro lado, quiçá em função de serem mais indesejáveis socialmente, os itens relativos a Maquiavelismo $(M=2,94 ; D P=0,54)$ e Psicopatia $(M=2,87 ; D P=$ $0,20)$ exigiram uma quantidade maior de traço latente, sendo os que apresentaram maior dificuldade, respectivamente, os itens 4 ("Costumo explorar outras pessoas para meu benefício próprio."; $\left.b_{4}=3,64\right)$ e o item 8 ("Eu costumo ser cínico."; $b_{4}=3,10$ ).

\section{Discussão}

Apesar da rápida profusão de estudos sobre a tríade sombria da personalidade, no Brasil, ainda são escassas as evidências em torno desse agrupamento de traços socialmente aversivos. Nessa direção, parece razoável, antes de qualquer tentativa de utilizar esses construtos conjuntamente, testar a adequação de medidas que os operacionalizem em contexto brasileiro. Sendo assim, considerando a popularização e pertinência de se utilizar medidas concisas para avaliar esses traços, decidiu-se conhecer os parâmetros da Dark Triad Dirty Dozen via Teoria de Resposta ao Item.

Destaca-se que o presente estudo reforçou as qualidades psicométricas dessa medida, com indicadores de ajuste que reforçam o modelo estrutural composto pelos três fatores em detrimento de um que representou a tríade sombria como um fator geral (Hair et al., 2009; Pilati \& Laros, 2007; Joanson \& Webster, 2010). Nesse ponto específico, cabe destacar que o presente estudo utilizou-se da análise fatorial confirmatória apenas para testar a unidimensionalidade do instrumento, fugindo do escopo testar modelos alternativos, como o bifator, que vem sendo reportado com os melhores indicadores de ajuste na literatura (Gouveia et al., 2016; Jonason et al., 2013; Jonason \& Luévano, 2013).

Verificou-se, ainda, a fidedignidade da medida, que se mostrou meritória, com valores do alfa de Cronbach e correlação inter-itens dentro dos padrões recomendados pela literatura $\left(\alpha>0,70 ; \mathrm{r}_{\text {mii }}>0,30\right.$; Clark \& Watson, 1995; Hair et al., 2009; Nunnaly, 1991). No que se refere à discriminação, esta ficou acima do ponto de corte que vem sendo proposto, sendo que os itens, em maioria, apresentaram alta discriminação (a $>1,70$; Baker, 2001), diferenciando adequadamente os participantes ao longo do traço latente avaliado. Comparando com os achados de Webster e Jonason (2013), verificou-se que os itens, nessa ocasião, foram mais discriminativos. Tais evidências somam-se às de Gouveia et al., (2016) que observaram, por meio da Teoria Clássica dos Testes, que todos os itens da DTDD foram discriminativos em contexto brasileiro.

No que tange ao parâmetro de dificuldade, assim como no estudo de Webster e Jonason (2013), os itens relativos ao Narcisismo foram os mais facilmente endossados, exigindo um valor mais baixo do traço latente para concordância total acerca do conteúdo do item. Não obstante, cabe destacar que a exceção foi o item 12, o mais difícil entre aqueles que compõem esse fator, sendo que este também apresentou maior dificuldade no estudo de Webster e Jonason. Em contrapartida, aqueles que formam Maquiavelismo e Psicopatia foram considerados mais difíceis, de modo que os limiares $b_{3}$ $\mathrm{e}_{4}$ exigiam um nível maior de theta. Um componente 
que pode explicar essa dificuldade pode ser o fato desses traços serem socialmente indesejados, portanto, o componente da desejabilidade social pode ter interferido na concordância aos itens. Logo, a partir desses resultados, pode-se pensar que, dos três componentes da tríade, talvez aqueles relativos ao narcisismo sejam os mais frequentes entre a população geral, quiçá sugerindo que este seja o traço menos sombrio da personalidade (Rauthman \& Kolar, 2012).

Apesar das contribuições advindas deste estudo, percebe-se que ele não está isento de limitações. Uma das restrições diz respeito a amostra utilizada, do tipo acidental, resultando, por exemplo, em um número desproporcional de mulheres neste estudo, compreendendo cerca de $70 \%$ dos participantes. Nessa direção, em possibilidades futuras, deve-se contar com amostras mais heterogêneas, inclusive distribuídas equitativamente quanto ao sexo, pois evidências empíricas vêm indicando que traços sombrios são mais frequentes em homens (Jonason \& Webster, 2010; Webster \& Jonason, 2013).

Ademais, outra limitação reside na natureza da medida empregada, do tipo lápis e papel. Nesse caso, em função do construto avaliado, percebe-se que os participantes podem ter falseado suas respostas, mostrando-se da melhor forma possível, como comentado anteriormente. Portanto, em possibilidades futuras, merece destaque controlar esse viés, seja aplicando uma medida de desejabilidade ou mesmo desenvolver formas mais acuradas de mensuração, que reduzem a possibilidade de falseamento das respostas, como pode ser o caso da utilização de medidas implícitas (Gouveia, Athayde, Mendes, \& Freire, 2012).

Apesar das limitações, avalia-se que os objetivos deste estudo foram alcançados, onde os resultados seguem a mesma direção daqueles encontrados por Webster e Jonason (2013), configurando-se como evidências adicionais dessa medida em contexto brasileiro. Dessa forma, com suporte da Teoria Clássica (Gouveia et al., 2016) e da Teoria de Resposta ao Item, percebe-se que a Dark Triad Dirty Dožen possui parâmetros psicométricos aceitáveis, sendo indicado o seu uso para fins de pesquisa, sobretudo quando são utilizadas múltiplas medidas e quando o pesquisador não dispõe de muito tempo para levar a cabo determinadas coletas. Por fim, indica-se que as possibilidades de estudo com essa medida não se esgotam aqui, cabendo futuramente verificar a sua estabilidade temporal, bem como conhecer em que medida ela se correlaciona com as versões mais longas.

\section{Referências}

Baker, F.B. (2001). The basics of item response theory. Washington, DC: ERIC Clearinghouse on Assessment and Evaluation.

Book, A., Visser, B. A., \& Volk, A. A. (2015). Unpacking "evil": Claiming the core of the dark triad. Personality and Individual Differences, 73, 29-38. doi: 10.1016/j.paid.2014.09.016

Browne, B. M., \& Cudeck, R. (1993). Alternative ways of assessing model fit. Em K. A. Bollen \& J. S. Long (Eds.), Testing structural equation models (pp. 136-162). Newbury Park, CA: Sage.

Carter, G. L., Campbell, A. C., \& Muncer, S. (2014). The dark triad personality: Attractiveness to women. Personality and Individual Differences, 56, 57-61. doi: $10.1016 /$ j.paid.2013.08.021

Clark, L. A., \& Watson, D. (1995). Constructing validity: Basic issues in objective scale development. Psychological Assessment, 7, 309-319. doi: 10.1037/1040-3590.7.3.309

Foster, J. D., \& Campbell, W. K. (2007). Are there such things as "Narcissists" in social psychology? A taxometric analysis of the Narcissistic Personality Inventory. Personality and Individual Differences, 43, 1321-1332. doi: 10.1016/j.paid.2007.04.003

Hair, J. F., Black, W. C., Babin, B. J., Anderson, R. E., \& Tatham, R. L. (2009). Análise multivariada de dados. Porto Alegre: Bookman.

Hu, L. T., \& Bentler, P. M. (1999). Cutoff criteria for fit indexes in covariance structure analisys: Conventional criteria versus new alternatives. Structural Equation Modeling, 6, 1-55. doi: 10.1080/10705519909540118

Huang, Y., \& Liang, C. (2014). A comparative study between the dark triad of personality and the big five. Canadian Social Science, 11, 93-98. doi. 10.3968/5715

Giammarco, E. A., \& Vernon, P. A. (2014). Vengeance and the dark triad: The role of empathy and perspective taking in trait forgiveness. Personality and Individual Differences, 67, 23-29. doi: 10.1016/j. paid.2014.02.010

Gouveia, V. V., Athayde, R. A. A., Mendes, L. A. C., \& Freire, S. E. A. (2012). Introdução às medidas implícitas: Conceitos, técnicas e contribuições. Diaphora, 12, 80-92. Recuperado de http://www. 
sprgs.org.br/diaphora/ojs/index.php/diaphora/ article/view/50/50

Gouveia, V. V., Monteiro, R. P., Gouveia, R. S. V., Athayde, R. A. A., \& Cavalcanti, T. M. (2016). Avaliando o lado sombrio da personalidade: Evidências psicométricas do Dark Triad Dirty Dozen. Interamerican Journal of Psychology, 50, 420-432. Recuperado de https://journal.sipsych.org/index.php/ IJP/article/view/126/pdf

Jakobwitz, S., \& Egan, V. (2006). The dark triad and normal personality traits. Personality and Individual Differences, 40, 331-339. doi: 10.1016/j. paid.2005.07.006

Jonason, P. K., Duineveld, J. J., \& Middleton, J. P. (2015). Pathology, pseudopathology, and the dark triad of personality. Personality and Individual Differences, 78, 43-47. doi: 10.1016/j.paid.2015.01.028

Jonason, P. K., Kaufman, S. B., Webster, G. D., \& Geher, G. (2013). What lies beneath the Dark Triad Dirty Dozen: Varied relations with the big five. Individual Differences Research, 11, 81-90. Recuperado de https:// faculty.newpaltz.edu/glenngeher/files/ jonason-et-al-2013.pdf

Jonason, P. K., Li, N. P., \& Buss, D. M. (2010). The coasts and benefits of the dark triad: Implications for mate proaching and mate retention tactics. Personaltiy and Individual Differences, 48, 373-378. doi: 10.1016/j.paid.2009.11.003

Jonason, P. K., \& Luévano, V. X. (2013). Walking the thin line between efficiency and accuracy: Validity and structural properties of the Dirty Dozen. Personality and Individual Differences, 55, 76-81. doi: 10.1016/j.paid.2013.02.010

Jonason, P. K., Li, N. P., Webster, G. D., \& Schmitt, D. P. (2009). The dark triad: Facilitating a short-term mating strategy in men. European Journal of Personality, 23, 5-18. doi: 10.1002/per.698

Jonason, P. K., \& Middleton, J. P. (2015). Dark triad: The "dark side" of human personality. Em J. D. Wright (Ed.), International Encyclopedia of the Social \& Behavioral Sciences (2a ed., v.5, pp. 671-675). Oxford: Elsevier.

Jonason, P. K., \& Webster, G. D. (2010). The dirty dozen: A concise measure of the dark triad. Psychological Assessment, 22, 420-432. doi: 10.1037/a0019265
Jones, D. N., \& Figueredo, A. J. (2013). The core of darkness: Uncovering the heart of the dark triad. European Journal of Psychology, 27, 521-531. doi: 10.1002/per.1893

Jones, D. N., \& Paulhus, D. L. (2011). Differentiating the Dark Triad within the interpersonal circumplex. Em L. M. Horowitz \& S. Strack, Handbook of interpersonal psychology: Theory, research, assessment, and therapeutic interventions (pp.249-269). New York: Wiley \& Sons.

Jones, D. N., \& Paulhus, D. L. (2014). Introducing the short dark triad (SD3): A brief measure of dark personality traits. Assessment, 21, 28-41. doi: 10.1177/1073191113514105

Kavanagh, P., James, S., Jonason, P. K., Chonody, J. M., \& Scrutton, H. E. (2014). The dark triad, schadenfreude, and sensational interests: Dark personality, dark emotions, and dark behaviors. Personality and Individual Differences, 68, 211-216. doi: 10.1016/j. paid.2014.04.020

LeBreton, J. M., Binning, J. F., \& Adorno, A. J. (2006). Subclinical psychopaths. Em C. J. Thomas, D. L. Segal \& M. Hersen (Eds), Comprehensive Handbook of Personality and Psychopathology: Personality and Everyday Functioning (Vol. 1, pp. 3-22). Hoboken, NJ: John Wiley \& Sons Inc.

Nunnally, J. C. (1991). Teoría psicométrica. México, DF: Trillas.

O’Boyle, E. H., Forsyth, D. R., Banks, G. C., Story, P. A., \& White, C. D. (2014). A meta-analytic test of redundancy and relative importance of the dark triad and five-factor model of personality. Journal of Personality. doi: 10.1111/jopy.12126.

Patrick, C. J., Fowles, D. C., \& Krueger, R. F. (2009). Triarchic conceptualization of psychopathy: Developmental origins of disinhibition, boldness, and meanness. Development and Psychopathology, 21, 913938. doi: 10.1017/S0954579409000492.

Paulhus, D. L. (2014). Toward taxonomy of the dark personalities. Current Directions in Psychological Science, 23, 421-426. doi: 10.1177/0963721414547737

Paulhus, D. L., \& Williams, K. M. (2002). The Dark Triad of personality: Narcissism, machiavellianism, and psychopathy. Journal of Research in Personality, 36, 556-563. doi: 10.1016/S0092-6566(02)00505-6 
Pilati, R., \& Laros, J. A. (2007). Modelos de equações estruturais em psicologia: Conceitos e aplicações. Psicologia: Teoria e Pesquisa, 23, 205-216. doi: 10.1590/S0102-37722007000200011

R Core Team (2015). R: A language and environment for statistical computing. $\mathrm{R}$ Foundation for Statistical Computing, Vienna, Austria. Recuperado de http://www.R-project.org/

Rauthmann, J. F., \& Kolar, G. P. (2012). How “dark" are the dark triad traits? Examining the perceived darkness of narcissism, Machiavellianism, and psychopathy. Personality and Individual Differences, 53, 884-889. doi: 10.1016/j.paid.2012.06.020

Rizopoulos, D. (2006). ltm: An R package for latent variable modelling and item response theory analyses. Journal of Statistical Software, 17, 1-25. Recuperado de http://www.jwalkonline.org/docs/ Grad\%20Classes/Spring\%2008/modmeas/ homework/ltm.pdf
Rosseel, Y. (2012). Lavaan: An R package for structural equation modeling. Journal of Statistical Software, 48, 1-36. doi: 10.18637/jss.v048.i02

Samejima, F. (1969). Estimation of latent ability using a response pattern of graded scores. Psychometrika Monograph Supplement No. 17. Richmond, VA: Psychometric Society.

Vernon, P. A., Villani, V. C., Vickers, L. C., \& Harris, J. A. (2008). A behavioral genetic investigation of the dark triad and the big 5. Personality and Individual Differences, 44, 445-452. doi: 10.1016/j. paid.2007.09.007

Webster, G. D., \& Jonason K. J. (2013). Putting the "IRT" in "Dirty": Item response theory analyses of the Dark Triad Dirty Dozen - An efficient measure of narcissism, psychopathy, and Machiavellianism. Personality and Individual Differences, 54, 302-306. doi: 10.1016/j.paid.2012.08.027

Recebido em: 18/02/2016

Reformulado em: 29/03/2016 Aceito em: 06/04/2016 
Sobre os autores:

Emerson Diógenes de Medeiros é psicólogo, mestre e doutor em Psicologia Social pela UFPB. Atualmente é professor adjunto do curso de graduação em Psicologia da Universidade Federal do Piauí (Campus de Parnaíba) e coordena o Laboratório de Avaliação Psicológica do Delta - LABAP. Seus interesses de pesquisa centram-se em Psicologia Social (valores humanos e atitudes), construção e adaptação de medidas psicológicas.

E-mail: emersondiogenes@gmail.com

Renan Pereira Monteiro possui formação em Psicologia pela Universidade Federal do Piauí, mestrado e doutorado em Psicologia Social pela Universidade Federal da Paraíba. Seus interesses de pesquisa centram-se no lado sombrio da personalidade (e.g., psicopatia, narcisismo, maquiavelismo e sadismo), Psicologia Social dos valores humanos, construção e adaptação de medidas psicológicas.

E-mail: renanpmonteiro@gmail.com

Rildésia Silva Veloso Gouveia é graduada em Psicologia pelo Centro Universitário de João Pessoa e doutora em Psicologia Social pela Universidade Federal da Paraíba. Atualmente é professora titular do Centro Universitário de João Pessoa. Alguns de seus interesses de pesquisa relacionam-se à avaliação de comportamentos antissociais e delitivos.

E-mail: rsvgouveia@gmail.com

Bruna da Silva Nascimento é graduada em Psicologia pela Universidade Federal do Piauí e mestre em Psicologia Social pela Universidade Federal da Paraíba. Atualmente é doutoranda em Psicologia pela University of Bath, Reino Unido. Seus interesses de pesquisa centram-se em Psicologia Evolucionista, relacionamentos íntimos e Psicologia Social (atitudes, valores humanos e preconceito).

E-mail: bruna.s.nascimento@hotmail.com

Valdiney Veloso Gouveia é licenciado (1989) e possui formação (2005) em Psicologia pela Universidade Federal da Paraíba, especialista em Psicometria (1993), mestre em Psicologia Social e do Trabalho (1991) pela Universidade de Brasília e doutor em Psicologia Social (1998) pela Universidade Complutense de Madri. Atualmente é professor titular na Universidade Federal da Paraíba.

E-mail:vvgouveia@gmail.com

Sobre os autores:

Prof. Emerson Diógenes de Medeiros

Universidade Federal do Piaú

Departamento de Psicologia

Laboratório de Avaliação Psicológica do Delta - LABAP

Av. São Sebastião, 2819

Parnaíba-PI, Brasil

CEP: 64202-020 